\title{
Originalien
}

Rechtsmedizin 2020 · 30:325-331 https://doi.org/10.1007/s00194-020-00404-1 Online publiziert: 5 . Juli 2020

(c) Springer Medizin Verlag GmbH, ein Teil von Springer Nature 2020

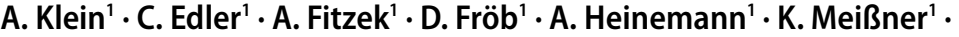

H. Mushumba' $\cdot$ K. Püschel ${ }^{\prime}$ A. S. Schröder' ${ }^{1}$ J. P. Sperhake' $\cdot$ F. Ishorst-Witte ${ }^{2}$. M. Aepfelbacher ${ }^{3} \cdot$ F. Heinrich

${ }^{1}$ Institut für Rechtsmedizin, Universitätsklinikum Hamburg-Eppendorf, Hamburg, Deutschland ${ }^{2}$ Bezirksamt Wandsbek, Hamburg, Deutschland

${ }^{3}$ Institut für Medizinische Mikrobiologie, Virologie und Hygiene, Universitätsklinikum HamburgEppendorf, Hamburg, Deutschland

\section{Der erste COVID-19-Hotspot in einer Hamburger Senioreneinrichtung}

\section{Präventionskonzept, Letalität und Obduktionsbefunde}

\section{Einleitung}

Der erste Coronavirus-Krankheit 2019 (COVID-19)-assoziierte Sterbefall eines deutschen Staatsangehörigen trat am 08.03.2020 ein. Es handelte sich um einen 59-jährigen, in Ägypten verstorbenen Mann aus Hamburg, der 12 Tage später im Hamburger Institut für Rechtsmedizin obduziert werden konnte [6]. Am 16.03.2020 verstarb der erste SARSCoronavirus-2 (SARS-CoV-2)-positive Bewohner einer Seniorenresidenz in Hamburg. Der 76-Jährige war mit dem neuen pandemischen Coronavirus infiziert, nach den Sektionsergebnissen jedoch nicht an diesem verstorben.

Nur wenige Tage später kam es zu einer ersten Cluster-Bildung in einem weiteren Pflegewohnheim desselben Stadtteils. In einer von 4 Pflegevillen, welche als geschützter, auf die Betreuung demenziell Erkrankter spezialisierter Wohnbereich angelegt ist, leben 60 Senioren. In diesem sog. Dementenhaus fand der erste pflegeheimbezogene Ausbruch der Hansestadt statt.

$\mathrm{Zu}$ diesem Zeitpunkt war vor dem Hintergrund der „Coronakrise“, auf Initiative des Leiters des Hamburger Instituts für Rechtsmedizin, bereits die systematische Evaluation aller SARSCoV-2-positiven Todesfälle mit der Behörde für Gesundheit und Verbraucher-

schutz (BGV) der Freien und Hansestadt Hamburg vereinbart worden. In Kooperation mit dem Institut für Medizinische Mikrobiologie, Virologie und Hygiene (UKE) wurde seitdem ein zentralisiertes Leichenschauverfahren für - ggf. mutmaßlich - COVID-19-Verstorbene umgesetzt. Diese Praxis wurde entgegen der ursprünglichen, mittlerweile öffentlich revidierten Empfehlungen des Robert Koch-Instituts (RKI) zu Leichenschau/Obduktion etabliert [14] und war in diesem Umfang international einzigartig.

Die vorliegende Arbeit legt das Vorgehen und die Besonderheiten bei der Bewältigung dieser erstmaligen „Hotspotproblematik“ in einer Pflegeeinrichtung Hamburgs dar - idealerweise als Hilfestellung in zu erwartenden, vergleichbaren Situationen. Ergänzt wird diese Darstellung durch die Ergebnisse autoptischer Untersuchungen des hier beschriebenen Kollektivs, um das Krankheitsbild COVID-19 näher zu charakterisieren.

\section{Methode}

Parallel zur Todesfallmeldung an das Gesundheitsamt durch den leichenschauenden Arzt wurden SARS-CoV-2-positive Sterbefälle in das Institut für Rechtmedizin transportiert. Bei Eingang eines Evaluationsfalles („Verdacht“/,,bestätigt") wurden (teils erneut) das zuständige Gesundheitsamt, die Pflegeeinrichtung sowie ggf. behandelnde Ärzte kontaktiert. Mittels Entnahme eines tiefen Nasen-/Rachenabstrichs (ESwab ${ }^{\circledR}$, Copan Italia, Brescia, Italien) bei allen aufgenommenen Verstorbenen im Institut gelang in den meisten Fällen innerhalb eines Tages eine Verifizierung der Coronainfektion. In allen Evaluationsfällen erfolgte die Anordnung der Sektion gemäß $\$ 25(4)$ des Infektionsschutzgesetzes (IfSG) durch das Gesundheitsamt. Die Evaluation beinhaltete, neben der Analyse klinischer Informationen, die autoptische sowie computertomographische und neuropathologische Untersuchung der Verstorbenen. Diese erfolgten unter empfohlenen Schutzvorkehrungen speziell für die Sektion von Coronatodesfällen sowie im allgemeinen Umgang mit infektiösem Gewebe [1,7]. Abweichend wurden durch die Hamburger Obduzenten FFP3-Masken (Feinstaubklassifizierung: 0,6 $\mu \mathrm{m} \geq 99 \%)$ statt N95Masken $(0,3 \mu \mathrm{m} \geq 95 \%)$ genutzt. Keiner der beteiligten Mitarbeiter im Institut für Rechtsmedizin in Hamburg entwickelte bis zur Einreichung dieses Manuskripts Symptome einer SARS-CoV-2-Infektion.

Nach Detektion einer Häufung von in der Rechtsmedizin eingegangenen Sterbefallmeldungen aus gegenständlicher 


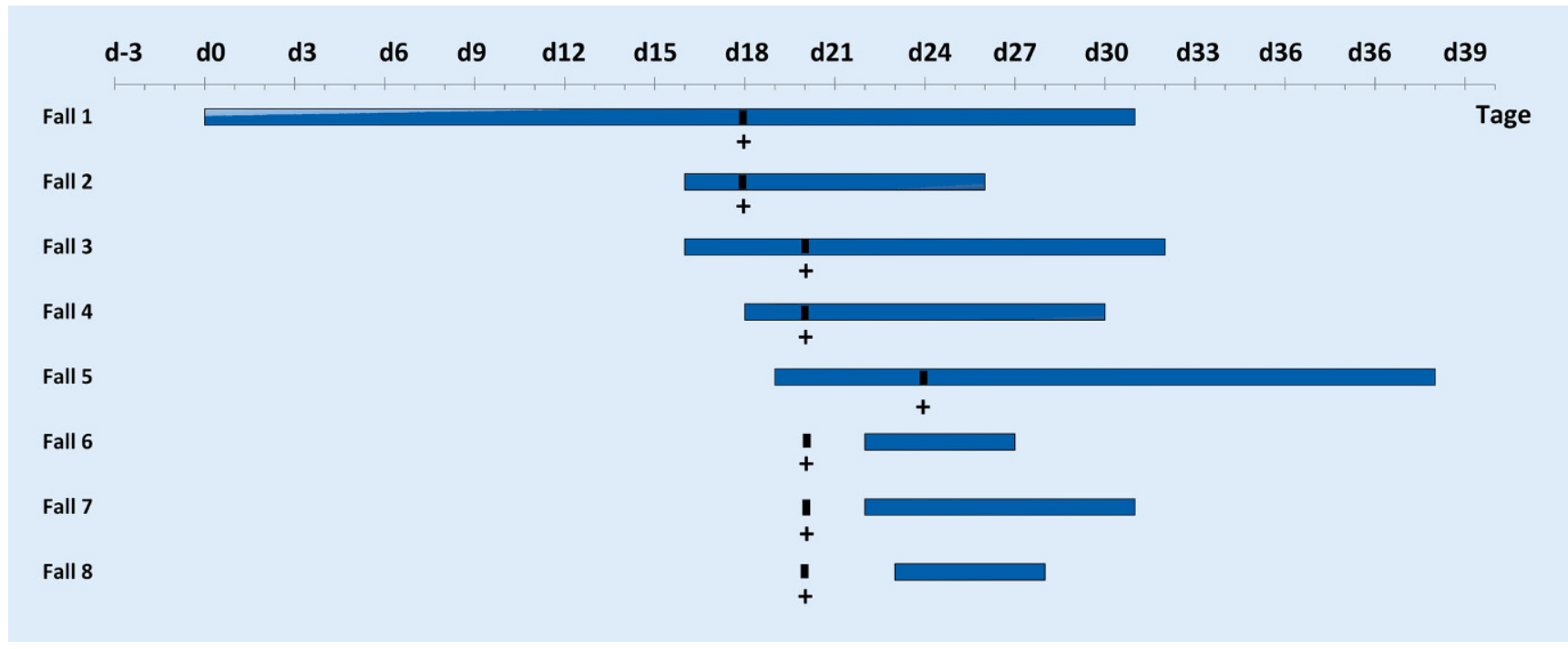

Abb. 1 D Darstellung des zeitlichen Symptomverlaufs bis zum Tod der verstorbenen Pflegeheimbewohner, inklusive Infektionsnachweis (Pluszeichen)

Seniorenresidenz fand ein Informationsaustausch zwischen ärztlichen Kollegen von Rechtsmedizin und Gesundheitsamt sowie Beteiligten der betroffenen Einrichtung statt. Teils wandten sich auch Angehörige verstorbener Bewohner an die Institutsleitung, um anamnestische Hintergründe mitzuteilen. Auf diesem Weg ließen sich relevante Faktoren wie Symptome, deren Beginn, Abläufe der behördlichen Testung, nachfolgende Maßnahmen und deren Umsetzung sowie ggf. ergänzende Angaben zu Vorerkrankungen (sofern nicht autoptisch belegt) erfassen. Eine detaillierte Auswertung sämtlicher Kranken- bzw. Pflegeakten war nicht möglich.

\section{Ergebnisse}

Anlässlich des dem Gesundheitsamt bekannt gewordenen gehäuften Auftretens der COVID-19-typischen Symptome (Fieber, trockener Husten) fanden zunächst symptombezogene, dann systematische - Testungen in der Seniorenresidenz statt (persönliche Mitteilung aus der zuständigen Behörde). Bei $65 \%$ der Bewohner $(n=39)$ bestätigte sich die Infektion. 17 der negativ Getesteten wurden in leerstehende Wohnbereiche einer anderen Einrichtung verbracht. Für verbliebene 4 negativ getestete Bewohner kam eine Verlegung aus individuellen, v. a. vorerkrankungsbedingten Gründen nicht in Betracht; sie konnten in ihren Einzelapartments isoliert werden. Anlässlich einer durch die BGV initiierten initialen notärztlichen Untersuchung fanden sich bei den positiv getesteten Senioren nur geringe Krankheitssymptome oder Beschwerden. Nach richterlicher Inaugenscheinnahme der Situation vor Ort wurde eine Türschließung der betroffenen Wohnbereiche angeordnet. Gestützt durch ein bereits bestehendes, generelles Besuchsverbot ließ sich so die räumliche Trennung bzw. Isolierung der Betroffenen realisieren. Unmittelbar nach Umzug der 17 negativ getesteten Bewohner wurde ein weiterer positiver Abstrich entnommen. Die Betroffene war aufgrund ihres schlechten Allgemeinzustands (COVID-typische Symptome) bereits initial isoliert worden; es bestand zu keinem Zeitpunkt ein Kontakt zu anderen Bewohnern. Nach Behördeneinschätzung war die Situation vor Ort beherrschbar. Die Infektionskette konnte jedoch bislang nicht im Einzelnen nachvollzogen werden. Demenzerkrankungsbedingt war eine gezielte Befragung der Bewohner nicht möglich. Eine Indexperson ließ sich nicht identifizieren. Nach Ablauf von 2 Wochen wurde für alle Bewohner des "Dementenhauses" die Quarantäne aufgehoben. Kontrollabstriche vor Rückzug in die Einrichtung zeigten einen weiteren Infektionsfall auf.
Im Rahmen des beschriebenen Ausbruchs verstarben insgesamt $20 \%(n=8)$ der SARS-CoV-2-infizierten Pflegeheimbewohner. In allen Fällen wurde autoptisch die Diagnose eines COVID19-assozierten Todes gestellt. Es handelte sich um 4 Frauen und 4 Männer im Alter von 77 bis 91 Jahren (mittleres Lebensalter: 86 Jahre). Der BMI lag bei den Frauen zwischen 17,2 und $22,8 \mathrm{~kg} / \mathrm{m}^{2}$, bei den Männern zwischen 19,9 und $25,6 \mathrm{~kg} / \mathrm{m}^{2}$ - es bestand somit Unter- bis Normalgewichtigkeit. Sechs der 8 COVID-19-Erkrankten verstarben in der Pflegeeinrichtung. Eine Bewohnerin und ein Bewohner verstarben nach 11- bzw. 12-tägigem Aufenthalt im Krankenhaus. $\mathrm{Zu}$ weiteren Todesfällen kam es bis zum Ende der Datenerhebung (28.04.2020) nicht (• Abb. 1).

Als führende initiale Symptome sind im gesamten Kollektiv unspezifische Allgemeinsymptome wie Abgeschlagenheit, Appetitlosigkeit sowie Dysphagie dokumentiert. Mehrheitlich konnte eine SARS-CoV-2-spezifische Symptomatik mit Fieber $(n=8)$ und Husten $(n=6)$ teils mit Würgereiz/Erbrechen $(n=2)$ eruiert werden; weiterhin traten Schnupfen sowie Diarrhö auf (je $n=1$ ). Vom ersten Auftreten der Symptomatik bis zum Versterben vergingen im Median 11 Tage (Spannweite 5 bis 31 Tage).

Todesursächlich war nach den Sektionsfeststellungen in allen 8 Fällen 
Rechtsmedizin 2020 · 30:325-331 https://doi.org/10.1007/s00194-020-00404-1

(c) Springer Medizin Verlag GmbH, ein Teil von Springer Nature 2020

\section{A. Klein · C. Edler · A. Fitzek · D. Fröb · A. Heinemann · K. Meißner · H. Mushumba · K. Püschel · A. S. Schröder · J. P. Sperhake · F. Ishorst-Witte · M. Aepfelbacher $\cdot$ F. Heinrich \\ Der erste COVID-19-Hotspot in einer Hamburger Senioreneinrichtung. Präventionskonzept, Letalität und Obduktionsbefunde}

\section{Zusammenfassung}

Hintergrund. Die durch das neue Coronavirus (SARS-CoV-2) bedingte Erkrankung Coronavirus-Krankheit 2019 (COVID-19) stellt eine Gefährdung für Ältere dar. Ende März 2020 kam es zu einem ersten, bislang größten Infektionsausbruch in einem Seniorenpflegewohnheim in Hamburg. Methode. Analyse von Verfahrensabläufen in einer von SARS-Coronavirus-2 (SARS-CoV-2) betroffenen Wohneinheit mit 60 demenziell vorerkrankten Senioren sowie umfassende Darstellung der Obduktionsbefunde SARSCoV-2-positiv getesteter dortiger Sterbefälle. Ergebnisse. In 39 von 60 Fällen infizierten sich Pflegeheimbewohner mit SARS-CoV-2. Eine Ausbreitung der Infektionswelle auf andere
Wohnbereiche ließ sich verhindern. In allen 8 Sterbefällen wurde durch die Obduktion die Diagnose eines COVID-19-bedingten Todes gestellt. Autoptisch fanden sich eine todesursächliche (Broncho-)Pneumonie sowie Korrelate relevanter kardialer, renaler und pulmonaler Vorerkrankungen in allen Fällen. In $75 \%(n=6)$ der Fälle waren frische Unterschenkelvenenthrombosen, in $66,7 \%(n=4)$ kombiniert mit peripheren Lungenarterienthromboembolien, nachweisbar.

Schlussfolgerung. Das im deutschsprachigen Raum erstmals systematisch beschriebene Kollektiv von SARS-CoV-2-Infizierten in einer Pflegeeinrichtung ist charakteristisch für die bislang bekannten klinischen und epidemiologischen Besonderheiten der neuen Coronainfektion. Durch die zentralisierte Evaluation aller Todesfälle in Hamburg war eine gezielte Aufarbeitung der SARS-CoV-2positiven Sterbefälle unter den betroffenen Heimbewohnern möglich. Der deutlich erhöhten Letalität von ca. $20 \%$ konnten in allen Fällen relevante Komorbiditäten multipler Organsysteme zugeordnet werden - die mit den klinischen Daten im Einklang standen.

Schlüsselwörter

SARS-CoV-2 · Geriatrie · Pflegeeinrichtung .

Infektionsweg · Letalität

\section{The first COVID-19 hotspot in a retirement home in Hamburg. Prevention concept, case fatality rate and post-mortem findings}

\section{Abstract}

Background. Coronavirus disease 2019 (COVID-19), a disease caused by the new coronavirus (SARS-CoV-2), is a particular threat to old people. At the end of March 2020, the first and so far largest outbreak of the disease occurred in a retirement home in Hamburg. Methods. Analysis of procedures in dealing with a residential unit affected by SARS-CoV-2, accommodating a risk group of 60 seniors with dementia is presented as well as a detailed presentation of post-mortem examination results of all 8 deceased tested positive for SARS-CoV-2.

Results. Out of 60 residents, 39 were infected by SARS-CoV-2. Due to preventive procedures it was possible to stop further spreading of the infection to other residential areas. In all 8 fatal cases, the autopsy diagnosis was death due to COVID-19. Autopsies revealed all COVID-19 patients to have a fatal (broncho)pneumonia and signs of relevant pre-existing cardiac, renal and pulmonary conditions in all cases. In $75 \%(n=6)$ of the cases a fresh venous thrombosis was found. In $66.7 \%(n=4)$ of the cases thrombotic events were combined with peripheral pulmonary artery thromboembolisms.

Conclusion. The cohort of SARS-CoV-2 infected residents of a nursing home is characteristic for clinical and epidemiological features of the new coronavirus disease. Due to a centralized evaluation of all fatalities at the Institute of Legal Medicine in Hamburg, a detailed examination of all deceased positive for SARS-CoV-2 was possible. Thereby, increased case fatality rates of approximately $20 \%$ could in all cases be assigned to a relevant number of pre-existing comorbidities of multiple organ systems, which was consistent with the clinical data available.

Keywords

SARS-CoV-2 - Geriatrics · Nursing home $\cdot$ Path of infection . Case fatality rate eine Pneumonie, mit unterschiedlich ausgeprägter bronchialer Beteiligung (Bronchopneumonie) und Zeichen eines generalisierten Entzündungsgeschehens (Milzauflockerung, allgemeine Gewebsbrüchigkeit und sog. Speckhautgerinnselbildungen), in einem Fall in Kombination mit einer subakuten Aortenklappenendokarditis. Betroffen waren stets beide Lungenflügel, bei teils mäßigem, zumeist massivem Ödem (bis zu einem Gesamtgewicht von $2005 \mathrm{~g}$ ), mit insgesamt ballonartig ausgerundetem Aspekt
(• Abb. 2a). Das Gewebe zeigte sich diffus verdichtet und zugleich allseits brüchig. Auf den Schnittflächen und teils subpleural fand sich eine scheckige bis mosaikartige, grau-rötliche bzw. von abgeblassten und hyperämischen Arealen durchsetzte Zeichnung (• Abb. 2b und 3) - dieser makroskopische Aspekt stellte sich in der Stärke seiner Ausprägung tendenziell proportional zur Erkrankungsdauer bzw. zur Dauer des intensivmedizinischen Supports dar. In $75 \%$ der Fälle ließen sich frische kleinkalibrige Wadenvenenthrombosen nachweisen $(n=6)$, bei $50 \%$ der Verstorbenen $(n=4)$ waren zudem akute, periphere Lungenarterienthromboembolien nachweisbar. Alle Verstorbenen wiesen Zeichen kardiovaskulärer, pulmonaler und renaler Komorbiditäten auf. Im Einzelnen fanden sich morphologische Korrelate für eine arterielle Hypertonie und eine chronische respiratorische Erkrankung (Emphysem, COPD) jeweils in allen Fällen, für eine chronische Herz- sowie Niereninsuffizi- 

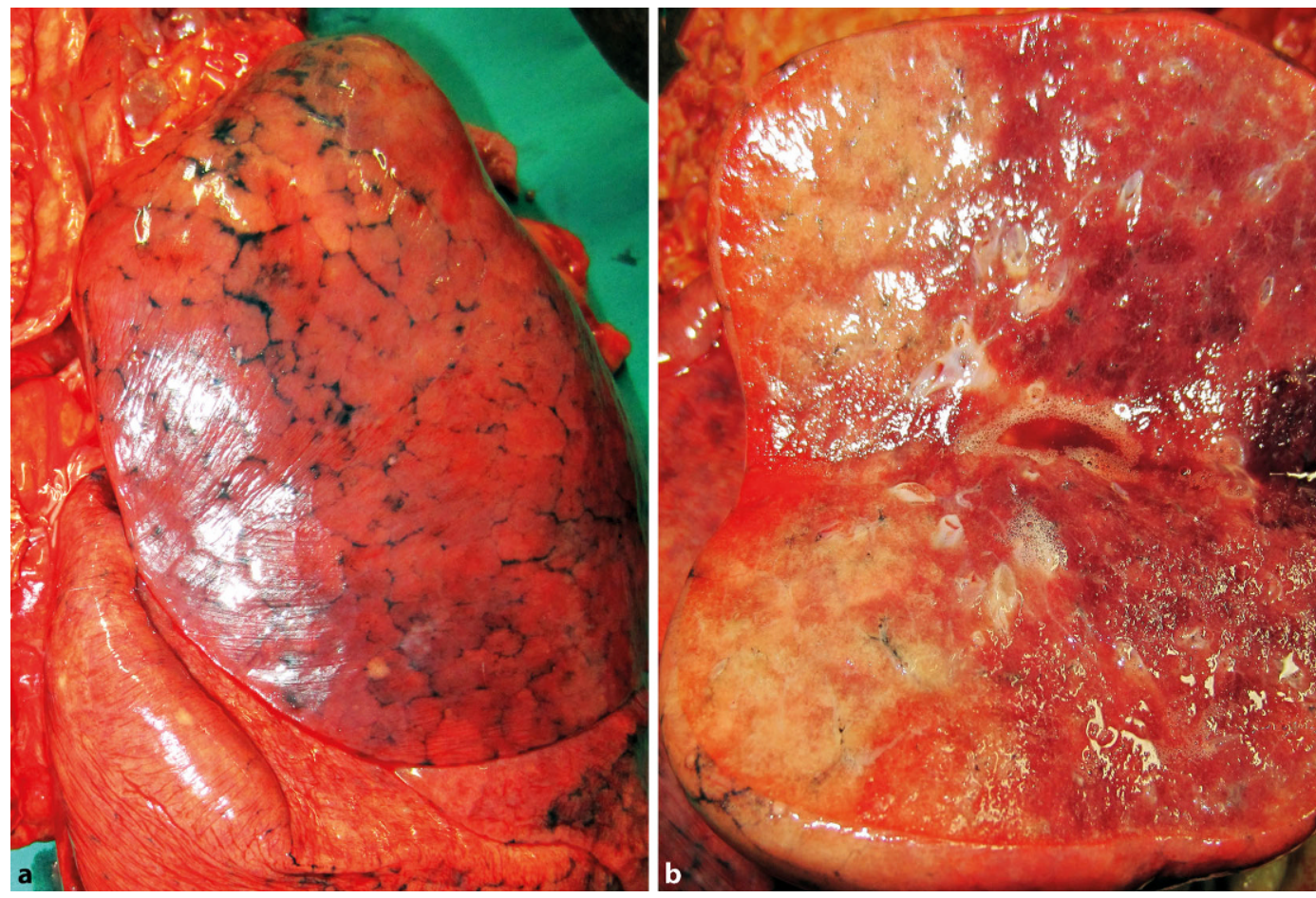

Abb. $\mathbf{2}<$ SARS-Coronavirus-2 (SARS-CoV-2)-typischer makroskopischer Lungenbefund eines balloniert imponierenden, schweren Lungenflügels (a) mit zugehöriger (ödematöser) Schnittfläche mit scheckiger Zeichnung (b)

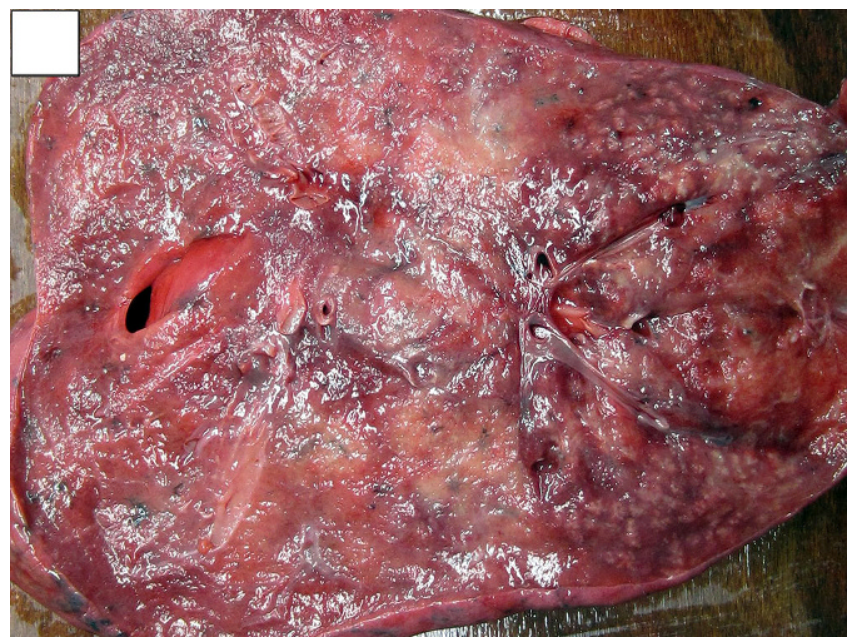

Abb. $3<$ Mosaikartiger Aspekt der Schnittfläche mit grob- und kleinfleckigem Wechsel blasser und hyperämischer Areale

enz in jeweils $87,5 \%$ der Fälle $(n=7)$. Diabetes-mellitus-assoziierte Pankreasveränderungen zeigten sich in 3 Fällen, bei einem Betroffenen kombiniert mit einer Leberverfettung (• Tab. 1).

Eine detaillierte Darstellung der Sektionsfeststellungen bzw. der makroskopischen und mikroskopischen Befunde ist Gegenstand von weiteren, kürzlich publizierten Studien [5, 18].

\section{Diskussion}

Die hier vorgelegte Studie beinhaltet $\mathrm{Da}$ ten aus der bislang wahrscheinlich welt- weit größten Obduktionsserie von SARSCoV-2-Infizierten. Insgesamt wurden in der Rechtsmedizin in Hamburg im März und im April 2020154 COVID-19Sterbefälle obduziert. Aufgrund eines bereits bei Auftreten des ersten COVID19-Todesfalles entwickelten Konzepts zur Erfassung und Untersuchung aller SARS-CoV-2-Verstorbenen in Hamburg gelang es frühzeitig, zahlreiche und wertvolle Informationen $\mathrm{zu}$ gewinnen - getreu dem Credo „Von den Toten lernen für die Lebenden“. Derzeit entsteht eine umfassende Datenbasis und Probensammlung wissenschaftli- chen Materials. Dies dient der Charakterisierung und dem Verständnis des Krankheitsbildes. So waren erhobene Sektionsbefunde (z. B. Thrombosen und Embolien) richtungweisend auch für therapeutische Ansätze (Anpassung der prophylaktischen Antikoagulation). Die Differenzierung zwischen Todesursachen mit und ohne Kausalität zu einer bestehenden SARS-CoV-2-Infektion ist gesundheitsstatistisch wie auch als Information für Angehörige von Bedeutung. Dem „Hamburger Weg“ folgen mittlerweile andere rechtsmedizinische und pathologische Institute [14].

Wie die Analyse der ersten erfassten positiv aufSARS-CoV-2-getesten Fälle in Hamburg ergab, starben etwa $62 \%$ der Erkrankten im krankenhäuslichen Setting $(n=48)$ und - in der Häufigkeit folgend $-25 \%$ in Pflegeheimen $(n=19)$.

Der in diesem Fall systematisch aufgearbeitete Pflegeheimausbruch zeigt einen typischen Ablauf eines solchen infektiologischen Geschehens. Während $75 \%$ der Verstorbenen eine SARS-CoV-2-spezifische Symptomatik (Kombination von Fieber und Husten) entsprechend den Kriterien des RKI aufwiesen [13], bestand bei allen eine unspezifische Allgemeinsymptomatik als Ausdruck ihrer Erkrankung. Nach einer Studie von Yang 


\begin{tabular}{|c|c|c|c|c|c|c|c|}
\hline Pat. & Lunge & Herz & Venen & Arterien & Nieren & Leber & Pankreas \\
\hline 1 & Pneumonie, LAE,COPD & $\begin{array}{l}\text { KHK, chron. Herzinsuffizienz, } \\
\text { Rechtsherzbelastung, Lipomato- } \\
\text { sis cordis }\end{array}$ & TVT & Arteriosklerose & $\begin{array}{l}\text { Chron. Nie- } \\
\text { reninsuffizi- } \\
\text { enz }\end{array}$ & Leberverfettung & Lipomatose \\
\hline 2 & $\begin{array}{l}\text { Pneumonie, LAE, COPD, } \\
\text { begl. Pleuritis, Anthra- } \\
\text { kose }\end{array}$ & $\begin{array}{l}\text { KHK, chron. Herzinsuffizienz, } \\
\text { Z. n. Myokardinfarkt, Lipomatosis } \\
\text { cordis }\end{array}$ & TVT & Arteriosklerose & $\begin{array}{l}\text { Chron. Nie- } \\
\text { reninsuffizi- } \\
\text { enz }\end{array}$ & $\begin{array}{l}\text { Leberverfettung, } \\
\text { chron. Blutstau- } \\
\text { ung, Hämangio- } \\
\text { me }\end{array}$ & - \\
\hline 3 & $\begin{array}{l}\text { Pneumonie, COPD, aku- } \\
\text { te Begleitbronchitis, } \\
\text { beginnende Pleuritis }\end{array}$ & $\begin{array}{l}\text { KHK, chron. Herzinsuffizienz, Z.n. } \\
\text { Myokardinfarkt, globale dilatative } \\
\text { Herzhypertrophie, Vorhofmyxom }\end{array}$ & - & Arteriosklerose & $\begin{array}{l}\text { Chron. Nie- } \\
\text { reninsuffizi- } \\
\text { enz }\end{array}$ & - & - \\
\hline 4 & $\begin{array}{l}\text { Pneumonie, COPD, } \\
\text { Anthrakose }\end{array}$ & $\begin{array}{l}\text { KHK, chron. Herzinsuffizienz, } \\
\text { biventrikuläre dilatative Herzhy- } \\
\text { pertrophie, Lipomatosis cordis }\end{array}$ & - & Arteriosklerose & $\begin{array}{l}\text { Chron. Nie- } \\
\text { reninsuffizi- } \\
\text { enz }\end{array}$ & - & - \\
\hline 5 & $\begin{array}{l}\text { Pneumonie, COPD, } \\
\text { akute Bronchitis }\end{array}$ & $\begin{array}{l}\text { KHK, chron. Herzinsuffizienz, Z.n. } \\
\text { Myokardinfarkt }\end{array}$ & TVT & Arteriosklerose & $\begin{array}{l}\text { Chron. Nie- } \\
\text { reninsuffi- } \\
\text { zienz, beg. } \\
\text { Schocknieren- } \\
\text { bildung }\end{array}$ & $\begin{array}{l}\text { Chron. Blutstau- } \\
\text { ung }\end{array}$ & Lipomatose \\
\hline 6 & $\begin{array}{l}\text { Pneumonie, LAE, COPD, } \\
\text { Anthrakose }\end{array}$ & $\begin{array}{l}\text { KHK, Z. n. Myokardinfarkt, Herz- } \\
\text { hypertrophie }\end{array}$ & TVT & Arteriosklerose & $\begin{array}{l}\text { Chron. Nie- } \\
\text { reninsuffizi- } \\
\text { enz, Nieren- } \\
\text { zysten }\end{array}$ & - & - \\
\hline 7 & $\begin{array}{l}\text { Pneumonie, COPD, } \\
\text { akute Begleitbronchitis }\end{array}$ & $\begin{array}{l}\text { KHK, chron. Herzinsuffizienz, } \\
\text { Aortenklappenendokarditis }\end{array}$ & TVT & Arteriosklerose & - & Schockleber & $\begin{array}{l}\text { Akut tryp- } \\
\text { tische Pan- } \\
\text { kreatitis }\end{array}$ \\
\hline 8 & $\begin{array}{l}\text { Pneumonie, LAE, COPD, } \\
\text { Pleuritis }\end{array}$ & KHK, chron. Herzinsuffizienz & TVT & Arteriosklerose & $\begin{array}{l}\text { Chron. Nie- } \\
\text { reninsuffizi- } \\
\text { enz }\end{array}$ & - & $\begin{array}{l}\text { Pankreas- } \\
\text { fibrose }\end{array}$ \\
\hline
\end{tabular}

et al. war unter später verstorbenen Patienten im Median eine Dauer von 11 Tagen zwischen Symptombeginn und der Notwendigkeit intensivmedizinischer Maßnahmen sowie eine Dauer von 18 Tagen zwischen Symptombeginn und dem Tod $\mathrm{zu}$ verzeichnen [19]. Dies ist mit einem in dieser Arbeit dokumentierten mittleren zeitlichen Intervall von 13,8 Tagen ( \pm 9,0 Tage) bis zum Tod vereinbar.

In besonderem Maß vorliegende, autoptisch dokumentierte kardiovaskuläre $(\text { HR } 1,31)^{1}$, pulmonale (HR 1,25) und renale Komorbiditäten (HR 1,19) im beschriebenen Kollektiv stimmen mit der Erfahrung überein, dass diese einen signifikanten Einfluss auf die Krankheitsschwere, indiziert durch ein ARDS [9, 17], sowie auf das Überleben von COVID-19-Patienten haben [3]. Auch Dreher et al. berichten in einer großen

1 Hazard ratio (Gefährdungsquote) des Kollektivs gegenüber dem Vergleichskollektiv zu versterben. aktuellen Studie über 50 hospitalisierte COVID-19-Patienten, die sämtlich die gleichen präexistenten Komorbiditäten aufwiesen [4]; einzig abweichend ließ sich unter den verstorbenen Senioren in keinem Fall Adipositas nachweisen, was bei dem betroffenen Kollektiv demenzerkrankter alter Menschen auch nicht zu erwarten ist.

Dass eine Pneumonie mit dem hieraus resultierenden respiratorischen Versagen bei COVID-19 zum Tod führt, ist hinlänglich bekannt. Diese war im hier untersuchten Kollektiv in allen Fällen mit einer begleitenden makroskopisch abgrenzbaren akuten Bronchitis vergesellschaftet, die sich auf eine hierfür ursächliche bakterielle Superinfektion zurückführen lässt, wie bereits durch Hanley und Osborn thematisiert [7, 11]. Zudem wurden erhöhte und persistierende Werte von Entzündungsparametern und D-Dimeren als prognostisch ungünstige klinische Prädiktoren für die Entwicklung des ARDS und einer Sepsis, hin- weisend für eine höhere intrahospitale Mortalität, eingeschätzt $[4,17,20]$. Als morphologisches Korrelat ließen sich identisch $\mathrm{zu}$ anderen bislang veröffentlichten Autopsiestudien [5, 8, 18] - in den hier vorgestellten Sektionsfällen Zeichen der Sepsis und akute Thrombosierungen identifizieren.

Sowohl die Komorbiditäten als auch der hier ermittelte deutlich höhere Altersdurchschnitt von 86 Jahren sind dem naturgemäßen Charakter einer Kohorte eines Seniorenheims zuzuordnen. Durch Onder et al. konnten anhand eines großen italienischen Patientenkollektivs ( $n=1625$ ) eine Häufung COVID19 -assozierter Sterbefälle in Patientenaltersgruppen $\geq 70$ Jahre, wie auch in unserer Arbeit aufgezeigt, festgestellt werden [10]. Zuletzt wurden Letalitätsraten von $6,96 \%$ für die Gesamtbevölkerung gezeigt. In Deutschland ist die Letalität von COVID-19 mit 3,94\% eher gering [15]. Altersstandardisierte Daten aus China zeigen eine 4,5- bis 9,5-fach 
erhöhte Letalität (6,4\% bzw. 13,4\%) in der $>60$ bzw. $>80$-jährigen Bevölkerung im Vergleich zur Gesamtpopulation [16]. Die Letalität in gegenständlich vorgestellter Fallserie war mit $20 \%$ deutlich erhöht - die Analyse umfasste jedoch ausschließlich Zugehörige der COVID19-Risikogruppe. Auch Boccardi et al. warnen vor COVID-19 als eine Erkrankung, die besonders oben besagtes, geriatrisches Kollektiv gefährdet [2] Vergleicht man die Sterbefallzahlen in der betroffenen Einrichtung im April 2020 (01.04.2020-28.04.2020) mit denen des entsprechenden Zeitraums der Vorjahre, bestätigt sich auch hier eine offensichtlich erhöhte COVID-19-assoziierte Mortalität (2020: $n=10$, zwei Bewohner verstarben ohne SARS-CoV2-Infektion; 2019: $n=1,2018: n=2$ ).

Sämtliche bisher veröffentliche Studienergebnisse beziehen sich auf Kollektive von Infizierten und/oder Patienten. Da nach unseren Kenntnissen eine auch nur annähernd vergleichbar große systematische Aufarbeitung von Obduktionsfällen COVID-19-Erkrankter außerhalb Hamburgs nicht existiert, fehlt (noch) die Grundlage für eine weiterführende vergleichende Analyse dieser Arbeit.

$\mathrm{Zu}$ vergleichbaren COVID-19-Ausbrüchen in Pflegeheimen ist es in Hamburg bis zum Zeitpunkt der Manuskripterstellung in weiteren 10 Fällen mit mindestens 29 Sterbefällen gekommen. Es ist eine dringende medizinische und gesellschaftliche Aufgabe, derartige Ausbrüche in Pflegeheimen mit einem präventiven Ansatz und in letzter Konsequenz mit einem klaren Ausbruchsmanagement zu verhindern bzw. einzudämmen [12].

Wir hoffen, dass die hier vorgestellten Untersuchungsabläufe und deren Ergebnisse für die Entwicklung weiterer und zukünftiger Bewältigungsstrategien in der Coronakrise helfen und Berücksichtigung finden können.

\section{Fazit für die Praxis}

\section{- Die COVID-19-assoziierte Letalität bei Pflegeheimbewohnern ist deutlich erhöht gegenüber Personen eines vergleichbaren Alterskollektivs. dargestellten Kohorte ein mittleres}

Alter von 86 Jahren und Komorbiditäten in allen Fällen auf.

- Todesursächlich war in allen Fällen eine Pneumonie.

- Eine demenzielle Erkrankung als Komorbidität erschwert die Rückverfolgung der Infektionskette und die Umsetzung von Isolationsmaßnahmen.

\section{Korrespondenzadresse}

\section{Dr. med. A. Klein}

Institut für Rechtsmedizin, Universitätsklinikum Hamburg-Eppendorf

Butenfeld 34, 22529 Hamburg, Deutschland anke.klein@uke.de

Danksagung. Wir danken allen an diesem Projekt beteiligten Mitarbeitern der hier aufgeführten, kooperierenden Institutionen - in besonderem Maße Frau Annegret Rothgänger für ihre inhaltliche Zuarbeit (Qualitätsmanagement Frank-WagnerHolding, Hanseatische Management $\mathrm{GmbH}$ ).

\section{Einhaltung ethischer Richtlinien}

Interessenkonflikt. A. Klein, C. Edler, A. Fitzek, D. Fröb, A. Heinemann, K. Meißner, H. Mushumba, K. Püschel, A.S. Schröder, J.P. Sperhake, F. Ishorst-Witte, M. Aepfelbacher und F. Heinrich geben an, dass kein Interessenkonflikt besteht.

Alle beschriebenen Untersuchungen am Menschen oder an menschlichem Gewebe wurden mit Zustimmung der zuständigen Ethik-Kommission (Antragsnummer: PV7311), im Einklang mit nationalem Recht sowie gemäß der Deklaration von Helsinki von 1975 (in der aktuellen, überarbeiteten Fassung) durchgeführt. Die Untersuchungen erfolgten unter Einhaltung der Vorgaben der Zentralen Ethikkommission der Bundesärztekammer.

\section{Literatur}

1. Barton LM, Duval EJ, Stroberg E, Gosh S, Mukhopadhyay S (2020) COVID-19 autopsies, Oklahoma, USA. Am J Clin Pathol 153(6):725-733

2. Boccardi V, Ruggiero C, Mecocci P (2020) COVID-19: a geriatric emergency. Geriatrics. https://doi.org/ 10.1007/s11357-020-00186-0

3. Docherty AB, Harrison EM, Green CA, Hardwick HE, Pius R, Norman L, Holden KA, Read JM, Dondelinger F, Carson G, Merson L, Lee J, Plotkin D, Sigfrid L, Halpin S, Jackson C, Gamble C, Horby PW, NguyenVan-Tam JS, Dunning J, Openshaw PJM, Baillie JK, Semple GM (2020) Features of 16,749 hospitalised UK patients with COVID-19 using the ISARIC WHO Clinical Characterisation Protocol. medRxiv. https://doi.org/10.1101/2020.04.23.20076042

4. Dreher $M$, Kersten A, Bickenbach J, Balfanz $P$, Hartmann B, Cornelissen C, Daher A, Stöhr R, Kleines M, Lemmen SW, Brokmann JC, Müller T, Müller-Wieland D, Marx G, Marx N (2020) The characteristics of 50 hospitalized COVID-19 patients with and without ARDS. Dtsch Arztebl Int 117:271-278

5. Edler C, Schröder AS, Aepfelbacher M, Fitzek AE, Heinemann A, Heinrich F, Klein A, Langenwalder F, Lütgehetmann M, Meißner K, Püschel K, Schädler J, Steurer S, Mushumba H, Sperhake JP (2020) Dying with SARS-CoV-2 infection - an autopsy study of the first consecutive 80 cases in Hamburg, Germany. Int J Legal Med 134:1275-1284. https:// doi.org/10.1007/s00414-020-02317-w

6. Fitzek A, Sperhake J, Edler C, Schröder AS, Heinemann A, Heinrich F, Ron A, Mushumba $\mathrm{H}$, Lütgehetmann M, Püschel K (2020) Evidence for systematic autopsies in COVID-19 positive deceased. Rechtsmedizin 30:184-189. https://doi. org/10.1007/s00194-020-00401-4

7. Hanley B, Lucas SB, Youd E, Swift B, Osborn M (2020) Autopsy in suspected COVID-19 cases. J Clin Pathol 73(5):239-242

8. Menter T, Hasbauer JD, Nienhold R, Savic $S$, Hopfer H, Deigendesch S, Frank S, Turek D, Willi N, Pargger H, Bassetti S, Leuppi JD, Cathomas G, Tolnay M, Mertz KD, Tzankov A (2020) Post-mortem examination of COVID19 patients reveals diffuse alveolar damage with severe capillary congestion and variegated findings of lungs and other organs suggesting vascular dysfunction. Histopathology. https://doi.org/10.1111/his.14134

9. Myers LC, Parodi SM, Escobar GJ, Liu VX (2020) Characteristics of hospitalized adults with COVID19 in an integrated health care system in California. JAMA. https://doi.org/10.1001/jama.2020.7202

10. Onder G, Rezza G, Brusaferro S (2020) Case-fatality rate and characteristics of patients dying in relation to COVID-19 in Italy. JAMA. https://doi.org/10. 1001/jama.2020.4683

11. Osborn M, Lucas S, Stewart R, Swift B, Youd E (2020) Autopsy practice relating to possible cases of COVID-19 (2019-nCov, novel coronavirus from China 2019/2020). https:// www.rcpath.org/uploads/assets/d5e28baf-57894b0f-acecfe370eee6223/fe8fa85a-f004-4a0c81ee4b2b9cd12cbf/Briefing-on-COVID-19autopsy-Feb-2020.pdf.Zugegriffen:26. Apr. 2020

12. Robert Koch-Institut (2020) Prävention und Management von COVID-19 in Altenund Pflegeeinrichtungen und Einrichtungen für Menschen mit Beeinträchtigungen und Behinderungen. https://www.rki.de/DE/ Content/InfAZ/N/Neuartiges_Coronavirus/ Alten_Pflegeeinrichtung_Empfehlung.html. Zugegriffen: 26. Apr. 2020

13. Robert Koch-Institut (2020) SARS-CoV-2 Steckbrief zur Coronavirus-Krankheit-2019 (COVID19). https://www.rki.de/DE/Content/InfAZ/N/ Neuartiges_Coronavirus/Steckbrief.html. Zugegriffen:26. Apr. 2020

14. Robert Koch-Institut (2020) Empfehlungen zum Umgang mit SARS-CoV-2-infizierten Verstorbenen. https://www.rki.de/DE/Content/InfAZ/N/ Neuartiges_Coronavirus/Verstorbene.html.Zugegriffen:26. Apr. 2020

15. Statista (2020) Letalitätsrate beim Coronavirus (COVID-19) in den am stärksten betroffenen Ländern. https://de.statista.com/statistik/daten/ studie/1103785/umfrage/mortalitaetsrate-descoronavirus-nach-laendern/.Zugegriffen: 26 . Apr. 2020

16. Verity R, Okell LC, Dorigatti I, Winskill P, Whittaker C, Imai N, Cuomo-Dannenburg G, Thompson $\mathrm{H}$, Walker P, Fu H, Dighe A, Griffin J, Cori A, Baguelin M, Bhatia S, Boonyasiri A, Cucunuba ZM, Fitzjohn R, Gaythorpe KAM, Green W, Hamlet A, Hinsley W, Laydon D, Nedjati-Gilani G, Riley S, van-Elsand S, 
Volz E, Wang H, Wang H, Xi X, Donnelly C, Ghani A, Ferguson N (2020) Estimates of the severity of coronavirus disease 2019: a model-based analysis. Lancet Infect Dis. https://doi.org/10.1016/S14733099(20)30243-7

17. Wang D, Hu B, Hu C, Zhu F, Liu X, Zhang J, Wang $B$, Xiang $H$, Cheng $Z$, Xiong $Y$, Zhao $Y$, Li $Y$, Wang X, Peng Z (2020) Clinical characteristics of 138 hospitalized patients with 2019 novel coronavirus-infected pneumonia in Wuhan, China. JAMA. https://doi.org/10.1001/jama.2020.1585

18. Wichmann $D$, Sperhake JP, Lütgehetmann $M$, Steurer $S$, Edler $C$, Heinemann A, Heinrich F, Mushumba H, Kniep I, Schröder AS, Burdelski C, de Heer G, Nierhaus A, Frings D, Pfefferle S, Becker H, Bredereke-Wiedling $H$, de Weerth $A$, Paschen HR, Sheikhzadeh-Eggers S, Stang A, Schmiedel S, Bokemeyer C, Addo MM, Aepfelbacher M, Püschel K, Kluge S (2020) Autopsy findings and venous thrombolism in patients with COVID-19: a prospective cohort study. Ann Intern Med. https:// doi.org/10.7326/M20-2003

19. Yang $X, Y u Y, X u J$, Shu $H$, Xia J, Liu $H, W u Y$, Zhang L, Yu Z, Fang M, Yu T, Wang Y, Pan S, Zou X, Yuan S, Shang Y (2020) Clinical course and outcomes of critically ill patients with SARS-CoV-2 pneumonia in Whuan, China: a single-centered, retrospective, observational study. Lancet Respir Med 8(5):475-481

20. Zhou F, Yu T, Du R, Fan G, Liu Y, Liu Z, Xiang J, Wang Y, Song B, Gu X, Guan L, Wei Y, Li H, Wu X, Xu J, Tu S, Zhang Y, Chen H, Cao B (2020) Clinical course and risk factors for mortality of adult inpatients with COVID-19 in Whuan, China: a retrospective cohort study. Lancet 395:1054-1062

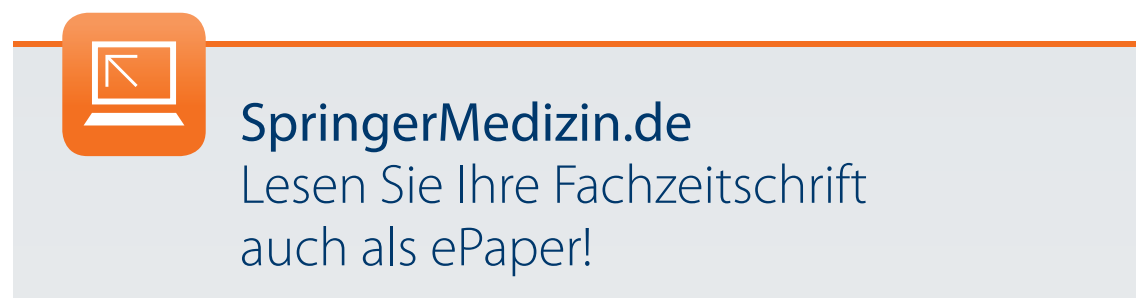

Als Abonnent können Sie Ihre Zeitschrift in verschiedenen Formaten lesen. Wählen Sie je nach Vorliebe und Situation aus, ob Sie die Zeitschrift als Print-Ausgabe, in Form von einzelnen Beiträgen auf springermedizin.de oder aber als komplette, elektronische ePaper-Ausgabe lesen möchten.

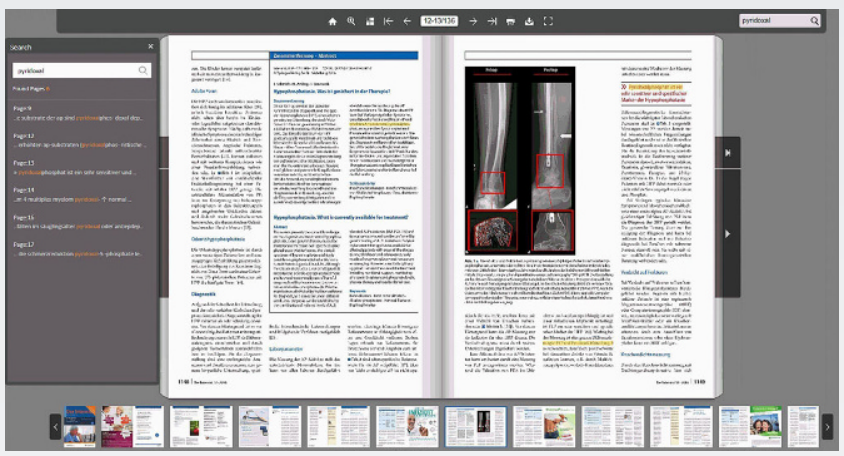

Die ePaper sind die identische Form der gedruckten Ausgaben. Sie sind nutzbar auf verschiedenen Endgeräten wie PC, Tablet oder Smartphone

Das sind die Vorteile des ePapers:

$>$ Das verlinkte Inhaltsverzeichnis führt Sie direkt zum gewünschten Beitrag

$>$ Eine Suchfunktion ermöglicht das Auffinden von Schlagworten innerhalb der Zeitschrift

> Jede Ausgabe kann als PDF heruntergeladen und damit auch offline gelesen werden bzw. auch gespeichert oder ausgedruckt werden.

> Als Abonnent haben Sie Zugang zu allen ePaper-Ausgaben ab 2016.

Sie finden die ePaper auf SpringerMedizin.de bei der jeweiligen Ausgabe Ihrer Fachzeitschrift. Klicken Sie auf den Button „Ausgabe als ePaper lesen“. 